\title{
Effects of mimicked acetylated HMGB1 on macrophages and dendritic cells
}

\author{
XIAOHONG $\mathrm{CHEN}^{1,2}$, YONG XU ${ }^{2}$, PING XIONG ${ }^{2}$, ZHENG TAN ${ }^{2}$, \\ FEILI GONG ${ }^{2}$, XIAOHUA HOU ${ }^{1}$ and FANG $\mathrm{ZHENG}^{2,3}$
}

\begin{abstract}
${ }^{1}$ Division of Gastroenterology, Union Hospital, Tongji Medical College, Huazhong University of Science and Technology, Wuhan, Hubei 430022; ${ }^{2}$ Department of Immunology, School of Basic Medicine, Tongji Medical College, Huazhong University of Science and Technology; ${ }^{3}$ Key Laboratory of Organ Transplantation, Ministry of Education, NHC, Chinese Academy of Medical Sciences, Wuhan, Hubei 430030, P.R. China
\end{abstract}

Received February 2, 2018; Accepted July 25, 2018

DOI: $10.3892 / \mathrm{mmr} .2018 .9584$

\begin{abstract}
Extracellular high mobility group box 1 (HMGB1) serves a critical role in inflammatory diseases. HMGB1 is released into the extracellular environment mainly by passive release from necrotic cells or active secretion from monocytes/macrophages following stimulation. However, the translocation of actively secreted HMGB1 from the nucleus to the cytoplasm with post-translational modifications such as acetylation is required; HMGB1 is then released into the extracellular space. Whether acetylation influences the extracellular function of HMGB1 remains unknown. In the present study, an optimized method of gene mutation by using well-designed primers in particular, which were employed to identify the mutant gene. The substitution of six lysine residues for glutamines was conducted to mimic acetylated HMGB1 (HMGB1-M) and observe the effects of HMGB1-M on macrophages and dendritic cells (DCs). Tumor necrosis factor (TNF)- $\alpha$ production in RAW 264.7 cells was assessed by ELISA. The phagocytic potential of RAW 264.7 cells, DC maturation and CXCR4 expression were analyzed by flow cytometry. The results of the present study revealed that HMGB1-M increased cytoplasmic translocation. Compared with HMGB1, HMGB1-M increased TNF- $\alpha$ production within RAW 264.7 cells and decreased the mean fluorescence intensity (MFI) of integrin $\alpha \mathrm{X}$, and the percentage and MFI of major histocompatibility complex-II on DCs. HMGB1-M exhibited no significant effects on phagocytosis of macrophages and expression frequency of cluster of differentiation 80 and chemokine receptor type 4 on DCs. These results
\end{abstract}

Correspondence to: Professor Fang Zheng, Department of Immunology, School of Basic Medicine, Tongji Medical College, Huazhong University of Science and Technology, 13 Hangkong Road, Wuhan, Hubei 430030, P.R. China

E-mail: zhengfangtj@hust.edu.cn

Key words: mutant, acetylated high mobility group box 1, inflammation, macrophage, dendritic cells suggested that HMGB1-M may partly promote inflammation and decrease DC maturation. Thus, the findings of the present study may provide insight into the complex role of HMGB1 in inflammatory diseases.

\section{Introduction}

High mobility group box 1 (HMGB1) is not only a non-histone nuclear protein that regulates gene transcription (1), but can also be passively released into the extracellular space as a proinflammatory mediator by necrotic cells, or in an active manner by lipopolysaccharide (LPS)-, tumor necrosis factor- $\alpha$ (TNF- $\alpha$ )-, interleukin- $1 \beta$ - and interferon- $\gamma$-stimulated monocytes or macrophages (2). The release of HMGB1 is regulated by elaborate mechanisms, including HMGB1 translocation from the nucleus to the cytoplasm with post-translational modifications of acetylation, methylation or phosphorylation, and its release into the extracellular environment with inflammasome activation upon appropriate stimulation $(3,4)$. When accumulating in the cytoplasm, HMGB1 has been reported to regulate autophagy (5). This evidence suggests that the function of HMGB1 is associated with its location. Previous studies have also reported that the extracellular functions of HMGB1 are dependent on its redox state and combination of target receptors, which mediates the responses to inflammation, immunity, chemotaxis and tissue regeneration (6-8). The biological roles of extracellular HMGB1 mainly involve its effects on macrophage proinflammatory function and dendritic cell (DC) antigen presentation (9,10). As extracellular HMGB1 is a critical mediator of lethality in inflammatory diseases (11), understanding the complex extracellular functions of HMGB1 is necessary for the development of potential treatments.

The acetylation of HMGB1 was first described in 1979, which revealed that there are two sites of acetylation in the HMGB1 protein, constituting lysine residues at positions 2 and 11 (12). A previous study indicated that acetylation of HMGB1 at lysine 2 (isolated from cells cultured in the presence of sodium n-butyrate) exhibited enhanced binding affinity to distorted DNA structures (13) and is required for their potential function in DNA replication (14). A recent study revealed via mass spectrometry, that 8 of 43 lysine residues 
are frequently modified in HMGB1 (15). The nuclear localization of HMGB1 is affected by lysine acetylation, particularly within the two major clusters of lysine residues at positions 27, 28 and 29 of the nuclear localization signal 1 (NLS1) and residues 181, 182 and 183 of NLS2 (15). It has been demonstrated that mutations of all six lysine residues into glutamines, as a mimic of acetyl-lysine within the two clusters, results in partial cytosolic localization (15). HMGB1 acetylation has been reported to regulate its interaction with DNA and repair DNA damage (16-18). In addition, accumulating experimental evidence has indicated that HMGB1 modified by acetylation serves an essential role in its nuclear translocation $(15,19)$; however, the effect of the post-translational modification of acetylation on the extracellular functions of HMGB1 is unknown. Therefore, the present study proposed that the extracellular functions of acetylated HMGB1 and non-acetylated HMGB1 differ.

In the present study, an optimized method of gene mutation was employed to obtain mimicked acetylated HMGB1 (HMGB1-M) and the effects of HMGB1-M and nonacetylated HMGB1 (HMGB1) on macrophages and DCs were compared.

\section{Materials and methods}

Animals. Six female 5-week-old C57BL/6 mice (18-20 g) were purchased from the Shanghai SLAC Laboratory Animal Co., Ltd. (Shanghai, China). All mice were housed in the Animal Laboratory Center of Tongji Medical College (Wuhan, China) in microisolator cages, at $22-23^{\circ} \mathrm{C}$ in $50-65 \%$ humidity under a 12:12 light-dark cycle and in specific pathogen-free conditions. The mice had access to a standard diet and water for one week prior to use in experiments. Animal studies were approved by the Animal Care and Use Committee of Tongji Medical College, HUST (Wuhan, China).

Construction of plasmids. The pET28a (Merck KGaA, Darmstadt, Germany)-HMGB1 plasmid (HMGB1 sequence from mouse) was used as a template to generate three lysine mutations at residues 27,28 , and 29 via site-directed mutagenesis by polymerase chain reaction (PCR) with mutant primer 1 . The mutant plasmids were then used as a template to generate another three mutated lysine residues 181, 182 and 183, again by site directed mutagenesis via PCR with mutant primer 2, forming the mutant plasmid (pET28a-HMGB1-M). Site directed mutagenesis PCR was performed using the Eppendorf PCR system (Eppendorf, Hamburg, Germany) with a final reaction volume of $50 \mu \mathrm{l}$ PCR mix containing $2 \mathrm{ng}$ template, all mutant primers at $0.3 \mu \mathrm{M}, 2512 \mathrm{X}$ PCR buffer, and each dNTP at $0.4 \mathrm{mM}$ and $1 \mathrm{U}$ KOD FX Taq DNA polymerase (Toyobo Life Science). The thermocycling conditions were as follows: Initial denaturation at $94^{\circ} \mathrm{C}$ for $2 \mathrm{~min}$ followed by 30 cycles, each at $98^{\circ} \mathrm{C}$ for $10 \mathrm{sec}$, annealing at $60{ }^{\circ} \mathrm{C}$ for $30 \mathrm{sec}$, extension at $68^{\circ} \mathrm{C}$ for $6 \mathrm{~min}$ $(1 \mathrm{kbp} / \mathrm{min})$. The PCR products were treated with the restriction enzyme, DpnI (Toyobo Life Science) at $37^{\circ} \mathrm{C}$ for $3 \mathrm{~h}$. A total of $100 \mu \mathrm{l}$ DH5 $\alpha$ competent cells (cat. no. C1100; Beijing Solarbio Science \& Technology Co., Ltd., Beijing, China) were transformed with $1 \mu \mathrm{l}$ of the aforementioned PCR products, according to the manufacturer's protocols and inoculated on Luria-Bertani plates containing kanamycin $(50 \mu \mathrm{g} / \mathrm{ml})$ at $37^{\circ} \mathrm{C}$ for $12 \mathrm{~h}$. A total of 10 random colonies were selected for screening mutants, and their plasmids were isolated by using the TIANprep Mini Plasmid kit (Tiangen Biotech Co., Ltd., Beijing, China), according to the manufacturer's protocols and PCR amplification was performed with the identifying primer. Identifying PCR was performed in a final volume of $20 \mu \mathrm{l}$ PCR mix containing $20 \mathrm{ng}$ template, each primer at $0.2 \mu \mathrm{M}, 10 \mu \mathrm{lmix}$ buffer and $2 \mathrm{U}$ Taq DNA polymerase (Thermo Fisher Scientific, Inc., Waltham, MA, USA) to identify the plasmids. The thermocycling conditions were as follows: Initial denaturation at $94^{\circ} \mathrm{C}$ for $5 \mathrm{~min}$ followed by 30 cycles, each at $94^{\circ} \mathrm{C}$ for $30 \mathrm{sec}$, annealing at $58^{\circ} \mathrm{C}$ for $30 \mathrm{sec}$, extension at $72^{\circ} \mathrm{C}$ for $1 \mathrm{~min}$ and a final extension at $72^{\circ} \mathrm{C}$ for $5 \mathrm{~min}$. The PCR products were evaluated by $1-2 \%$ agarose gel electrophoresis and the bands were visualized using GoldView I Nuclear Staining Dyes (Beijing Solarbio Science \& Technology Co., Ltd.). Subsequently, the pET28a-HMGB1 and pET28a-HMGB1-M plasmids were employed for PCR amplification with transferring primers, according to the identifying PCR conditions above. The PCR products and pEGFP-N1 plasmid (Clontech; Takara Bio, Inc., Otsu, Japan) were digested with XhoI (New England BioLabs, Inc., Ipswich, MA, USA) and BamHI (New England BioLabs, Inc.), then the PCR products and pEGFP-N1 plasmid were linked with T4 DNA Ligase (New England BioLabs, Inc.), according to the manufacturer's protocols. Reconstructed pEGFP-N1-HMGB1 and pEGFP-N1-HMGB1-M plasmids were first screened by PCR with transferring primers according to the identifying PCR conditions above, and double enzyme digestion identification was performed with XhoI and BamHI. All constructs were immediately sent to company (Thermo Fisher Scientific, Inc.) for direct DNA sequencing. The coding sequence of mouse HMGB1 (GenBank: BC006586.1; https:/www.ncbi.nlm.nih .gov/genbank/) was used for sequencing blast (https://blast .ncbi.nlm.nih.gov/Blast.cgi).

The primers used were: Mutant primer 1, HMGB1, forward 5'-CAGCAGCAGCACCCGGATGCTTCTGTCAAC-3', reverse 5'-TGCTGCTGCTGGTGCTCCTCCCGGCAAG-3'; mutant primer 2, HMGB1, forward 5'-AGCCAGCAACAGAAGGAA GAGGAAGATGATGAG-3', reverse, 5'-CTTCTGTTGCTG GCTCTTTTCAGCCTTGAC-3'; identifying primer 1, forward 5'-GGGAGGAGCACCAGCAGC-3', reverse 5'-CAGGATGCT CGCCTTTGATT-3'; identifying primer 2, forward 5'-GCCGGG AGGAGCACCAGC-3', reverse 5'-TCATCTTCCTCTTCCTGC TGTTG-3' and transferring primer, forward 5'-CCCTCGAGA TGGGCAAAGGAGATCCTAAGA-3' and reverse, 5'-CGG GATCCCGTTCATCATCATCATCTTCTTCTTCA-3'. The nucleotides in bold font indicate mutant sites.

Expression and purification of recombinant $H M G B 1$ and HMGB1-M. The pET28a-HMGB1 and pET28a-HMGB1-M plasmids $(50 \mathrm{ng})$ were transformed into $100 \mu \mathrm{l}$ protease-deficient Escherichia coli, strain BL21 (Beijing Solarbio Science $\&$ Technology Co., Ltd.) at $42{ }^{\circ} \mathrm{C}$ for $90 \mathrm{sec}$, according to the DH5 $\alpha$ transformation protocol, The bacteria were incubated in ZYP-5052 medium (Amresco, LLC, Solon, OH, USA) containing kanamycin $(50 \mu \mathrm{g} / \mathrm{ml})$ overnight at $37^{\circ} \mathrm{C}$ with vigorous agitation. The bacterial solution was centrifuged at $4^{\circ} \mathrm{C}, 8,000 \mathrm{x} \mathrm{g}$ for $10 \mathrm{~min}$. Then, cell lysis and purification of the recombinant protein were performed with $1 \mathrm{ml}$ HisTrap 
FF Crude columns (GE Healthcare Life Sciences, Little Chalfont, UK), according to the manufacturer's protocols. The affinity-purified recombinant proteins were further desalted using Zeba Spin Desalting Columns (Pierce; Thermo Fisher Scientific, Inc.), according to the manufacturer's protocols. The recombinant proteins were further purified by filtration and stored in aliquots at $-80^{\circ} \mathrm{C}$ until use. Contaminating lipolysaccharide (LPS from protein preparations) was detected by a Pierce LAL Chromogenic Endotoxin Quantitation kit (Thermo Fisher Scientific, Inc.), according to the manufacturer's protocols. The LPS content in purified HMGB1 and HMGB1-M samples was 1.31 and $1.11 \mathrm{pg} / \mu 1$, respectively. For all DC or RAW264.7 stimulation experiments with the recombinant proteins, polymyxin B (Sigma-Aldrich; Merck KGaA) was added to the cell culture medium at 6 units of polymyxin B per picogram of LPS. Proteins were detected by Coomassie blue staining at room temperature overnight following SDS-PAGE gel and protein purity was determined using BandScan software version 5.0 (Glyko, Novato, CA, USA).

Western blot analysis. Equal volumes (4 $\mu \mathrm{l})$ of purified HMGB1 $(0.32 \mu \mathrm{g} / \mu \mathrm{l})$ or HMGB1-M $(0.19 \mu \mathrm{g} / \mu \mathrm{l})$ proteins were separated by $12 \%$ SDS-PAGE. The protein concentration was determined in duplicate wells with a BCA protein assay kit (Thermo Fisher Scientific, Inc.), according to the manufacturer's protocols. Proteins were transferred to a $0.45-\mu \mathrm{m}$ polyvinylidene difluoride membrane, the membranes were incubated with $5 \%$ nonfat dry milk for $2 \mathrm{~h}$ at room temperature. Then, blots were incubated with rabbit monoclonal anti-HMGB1 (1:10,000; cat. no. ab79823, Abcam, Cambridge, MA, USA) overnight at $4^{\circ} \mathrm{C}$. The protein bands were visualized with Pierce ${ }^{\mathrm{TM}}$ ECL Plus Western Blotting Substrate (Thermo Fisher Scientific, Inc.), according to the manufacturer's protocols following incubation with goat anti-rabbit IgG-horseradish peroxidase-conjugated secondary antibodies (1:5,000; cat. no. sc-2004; Santa Cruz Biotechnology, Inc. Dallas, TX, USA) for $1 \mathrm{~h}$ at room temperature.

Transfection of the recombinant plasmids into cells and GFP imaging. RAW 264.7 cells (American Type Culture Collection, Manassas, VA, USA) were cultured in Dulbecco's modified Eagle's medium (DMEM; HyClone; GE Healthcare) with $10 \%$ heat-inactivated fetal bovine serum (FBS; Gibco; Thermo Fisher Scientific, Inc.) in a humidified $5 \% \mathrm{CO}_{2}$ incubator at $37^{\circ} \mathrm{C}$. The day prior to transfection, $5 \times 10^{5}$ cells were plated on glass coverslips in 24 -well plates. When $90 \%$ confluence was achieved, cells were transfected with $1 \mu \mathrm{g}$ pEGFP-N1, pEGFP-N1-HMGB1, or pEGFP-N1-HMGB1-M plasmids, respectively using GenePORTER reagent (Genlantis, San Diego, CA, USA) according to the manufacturer's protocols; untransfected cells served as the negative control. After $48 \mathrm{~h}$ following transfection, cells were washed twice with ice-cold PBS. Then, cells were fixed in $4 \%$ paraformaldehyde at room temperature for $30 \mathrm{~min}$ and incubated with DAPI (Invitrogen; Thermo Fisher Scientific, Inc.) at room temperature for 5 min to stain the cell nuclei. Images were captured at $x 400$ magnification using an inverted fluorescence microscope (CKX41SF; Olympus Corporation, Tokyo, Japan) with an excitation wavelength of $488 \mathrm{~nm}$, and the percentage of cells with HMGB1 cytoplasmic accumulation was manually counted under several randomly selected microscopic fields. Cell fluorescence intensity was quantified with ImageJ version 1.4 (National Institutes of Health, Bethesda, MD, USA).

ELISA and phagocytosis assay. A total of 5x10 RAW 264.7 cells were cultured in 24-well chamber slides in DMEM supplemented with 10\% FBS and stimulated with HMGB1-M $(5 \mu \mathrm{g} / \mathrm{ml})$ or HMGB1 $(5 \mu \mathrm{g} / \mathrm{ml})$ recombinant protein for $16 \mathrm{~h}$ to induce TNF- $\alpha$ secretion in a humidified $5 \% \mathrm{CO}_{2}$ incubator at $37^{\circ} \mathrm{C}$; untreated cells served as the negative control (MED group). Culture supernatants were collected and assayed for TNF- $\alpha$ production with an ELISA kit (cat. no. 88-7324-88; Invitrogen; Thermo Fisher Scientific, Inc.), according to the manufacturer's protocols. Then, the cells were washed twice with PBS and incubated with fluorescein isothiocyanate (FITC)-dextran (1,000 $\mu \mathrm{g} / \mathrm{ml}$; Sigma-Aldrich; Merck KGaA) for $2 \mathrm{~h}$ in a $5 \% \mathrm{CO}_{2}$ incubator at $37^{\circ} \mathrm{C}$. Cells were fixed in $4 \%$ paraformaldehyde at room temperature for $30 \mathrm{~min}$ and then incubated with DAPI (Invitrogen; Thermo Fisher Scientific, Inc.) at room temperature for $5 \mathrm{~min}$ to stain the cell nuclei. Images were captured from ten randomly selected microscopic fields at magnification $\mathrm{x} 400 \mathrm{using}$ an inverted fluorescence microscope (CKX41SF, Olympus Corporation) with an excitation wavelength of $488 \mathrm{~nm}$. Cells were also assessed by flow cytometry (LSR II; BD Biosciences) and analyzed using FlowJo software version 7.6.1 (Tree Star Inc., Ashland, OR, USA). The $\%$ phagocytosis was the $\%$ FITC $^{+}$cells of total mononuclear cells.

Treatment of bone marrow-derived DCs (BMDCs) and flow cytometry. Mice were sacrificed and disinfected in $75 \%$ ethanol for $5 \mathrm{~min}$. The femur and tibia were harvested to acquire BMDCs from mouse bone marrow, as described previously (20). After 7 days of culture in RPMI 1640 medium (Gibco; Thermo Fisher Scientific, Inc.) supplemented with $10 \% \mathrm{FBS}$ in a $5 \% \mathrm{CO}_{2}$ incubator at $37^{\circ} \mathrm{C}, 1 \times 10^{6}$ cells were stimulated with HMGB1 $(5 \mu \mathrm{g} / \mathrm{ml})$ or HMGB1-M $(5 \mu \mathrm{g} / \mathrm{ml})$ for $48 \mathrm{~h}$ and analyzed with flow cytometry; untreated cells (MED group) served as the negative control. The cells were incubated with monoclonal antibodies (mAbs) for $30 \mathrm{~min}$ at room temperature, and the fluorochrome-conjugated mAbs (BD Biosciences, Franklin Lakes, NJ, USA) used were as follows: Integrin $\alpha \mathrm{X}$ (CD11c)-phycoerythrin (PE)-Cy7, cluster of differentiation (CD)80-FITC, major histocompatibility complex (MHC)-II-allophycocyanin (eBioscience; Thermo Fisher Scientific, Inc.), and chemokine receptor type 4-PE (CXCR4; BD Biosciences). Cells were acquired on a flow cytometer (LSR II; BD Biosciences) and the data was analyzed using FlowJo software version 7.6.1 (Tree Star Inc.).

Statistical analysis. Data are presented as the mean \pm standard error of the mean of two or three independent experiments. Statistical analysis was performed using GraphPad Prism version 5.0 (GraphPad Software, Inc., La Jolla, CA, USA). The difference between two groups was performed using two-tailed unpaired Student's t-test. Comparisons between multiple groups were performed with one-way analysis of variance and a Newman-Keuls post-hoc test. $\mathrm{P}<0.05$ was considered to indicate a statistically significant difference. 

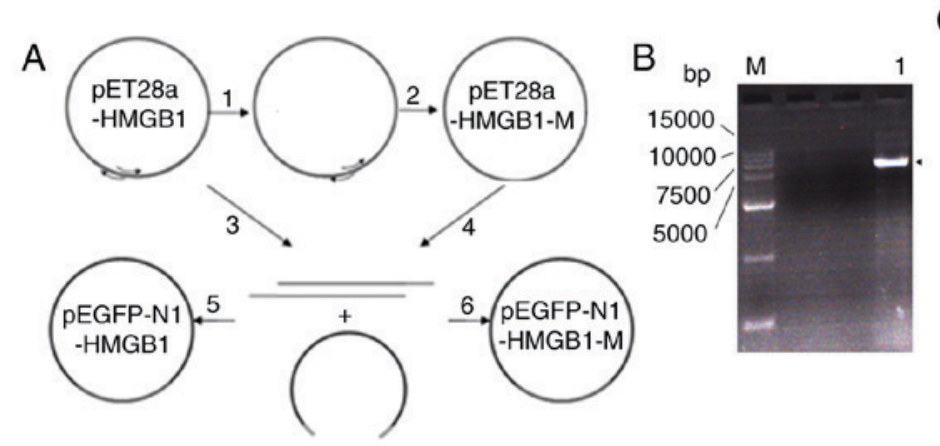

C $\begin{array}{lllllllllllll}1 & 2 & 3 & 4 & 5 & 6 & 7 & 8 & 9 & 10 & 11 & \mathrm{M}\end{array}$
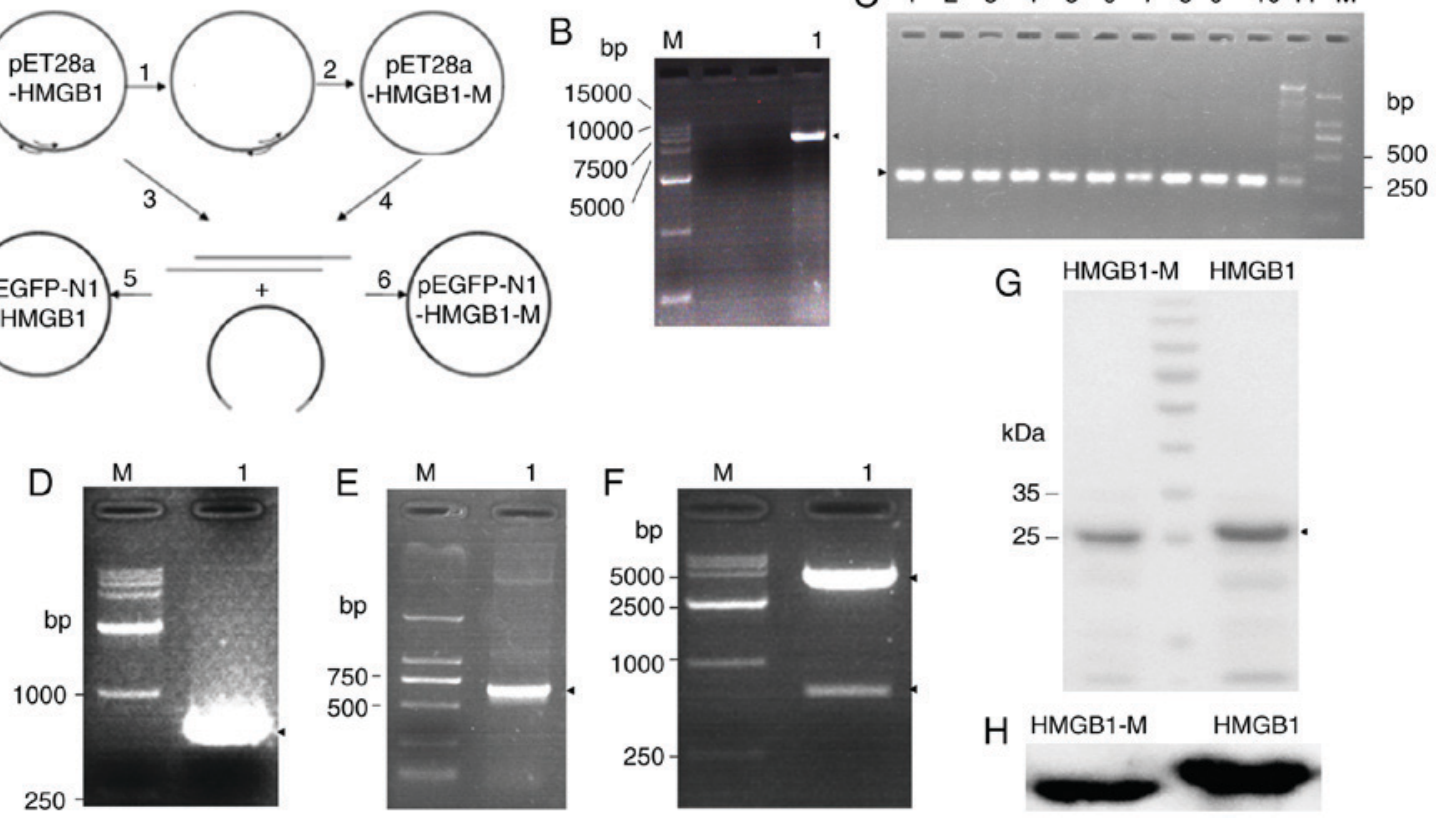

Figure 1. Mutation and reconstruction of HMGB1 and HMGB1-M expression plasmids, and the verification of purified HMGB1-M and HMGB1 proteins in Escherichia coli. (A) Schematic for the mutation and reconstruction of the HMGB1 expression plasmid. (B) Agarose gel electrophoresis of the products of mutagenesis PCR. (C) Plasmid PCR method to distinguish the desired mutant from the wild-type plasmid, lanes 1-10: Plasmid to be detected as template and lane 11: Wild-type plasmid as the control. (D) Amplified PCR product of HMGB1-M. (E) Plasmid PCR method to confirm the HMGB1-M gene insertion into the vector of pEGFP-N1. (F) Double restriction enzyme digestion of the desired plasmid. (G) HMGB1 protein samples with loading buffer were separated by 12\% SDS-PAGE and visualized by Coomassie blue staining. (H) Proteins were identified by western blot analysis using monoclonal anti-HMGB1 antibodies. Arrows indicate target gene or protein. HMGB1, high mobility group box 1; HMGB1-M, mimicked acetylated HMGB1; M, DNA or protein marker; PCR, polymerase chain reaction.

\section{Results}

Preparation of HMGB1 and HMGB1-M expression plasmids and proteins. The pET28a-HMGB1 plasmid was prepared in the present study; other plasmids were reconstructed according as presented in the flowchart (Fig. 1A). The pET28a-HMGB plasmid was used as template for PCR amplification with the mutant primer 1 to acquire partly mutant HMGB1 by mutating lysine into glutamine at positions 27, 28 and 29. The PCR-amplified circular products ( $\sim 6 \mathrm{~kb})$ were verified by agarose gel electrophoresis (Fig. 1B). In the present study, the aforementioned circular PCR products were transfected into DH5 $\alpha$ competent cells; 10 random transformed colonies were selected to screen for mutants using PCR amplification with identifying primer 1, which was designed against the mutant gene but not the wild-type gene. The mutant samples only had one bright band (285 bp), whereas the wild-type sample had multiple bands (Fig. 1C). Subsequently, one of the mutated plasmids was used as a template for amplification with mutant primer 2 to acquire mutant HMGB1 via the mutation of lysine residues 181, 182 and 183 into glutamines as aforementioned. Random colonies were selected for screening mutants to perform PCR amplification with identifying primer 2 as described above (data not shown). Following sequencing analysis of the plasmids, the pET28a-HMGB1-M plasmid was successfully constructed (data not shown).

In the present study, two eukaryotic expression plasmids were reconstructed by transferring HMGB1-M and HMGB1 DNA fragments from pET28a to pEGFP-N1 plasmids by gene recombination. Using the pET28a-HMGB1-M plasmid as a template, a HMGB1-M DNA fragment was acquired by PCR amplification with transferring primers. PCR products (648 bp) were verified by agarose gel electrophoresis (Fig. 1D). Then, PCR products were cloned into pEGFP-N1 plasmids by double enzyme digestion and ligation reactions. Following the transformation of a BL21 strain with the reaction mixtures, random colonies were selected to acquire plasmids. All plasmids were verified by PCR (Fig. 1E) and restriction enzyme digestion (Fig. 1F). The reconstruction process of the pEGFP-N1-HMGB1 plasmid was conducted in the same manner as that for the pEGFP-N1-HMGB1-M plasmid (data not shown). The pEGFP-N1-HMGB1-M/HMGB1 plasmids were finally confirmed by sequencing analysis (data not shown).

To investigate the extracellular functions of the HMGB1-M protein, pET28a-HMGB1-M/HMGB1-transformed strains were employed to induce protein expression and the target proteins were then purified. HMGB1 and HMGB1-M proteins were $>80 \%$ pure, as revealed by Coomassie blue staining of the SDS-PAGE gel (Fig. 1G). In addition, the purified protein was identified by western blot analysis (Fig. 1H). These results indicated that HMGB1 and HMGB1-M protein were successfully acquired.

HMGB1-M is expressed in the nucleus and cytoplasm of RAW 264.7 cells. The HMGB1-M-GFP plasmid was transfected into RAW 264.7 cells to observe the intracellular distribution pattern of this protein. HMGB1-M was expressed in the nucleus and cytoplasm, while HMGB1 was concentrated in 

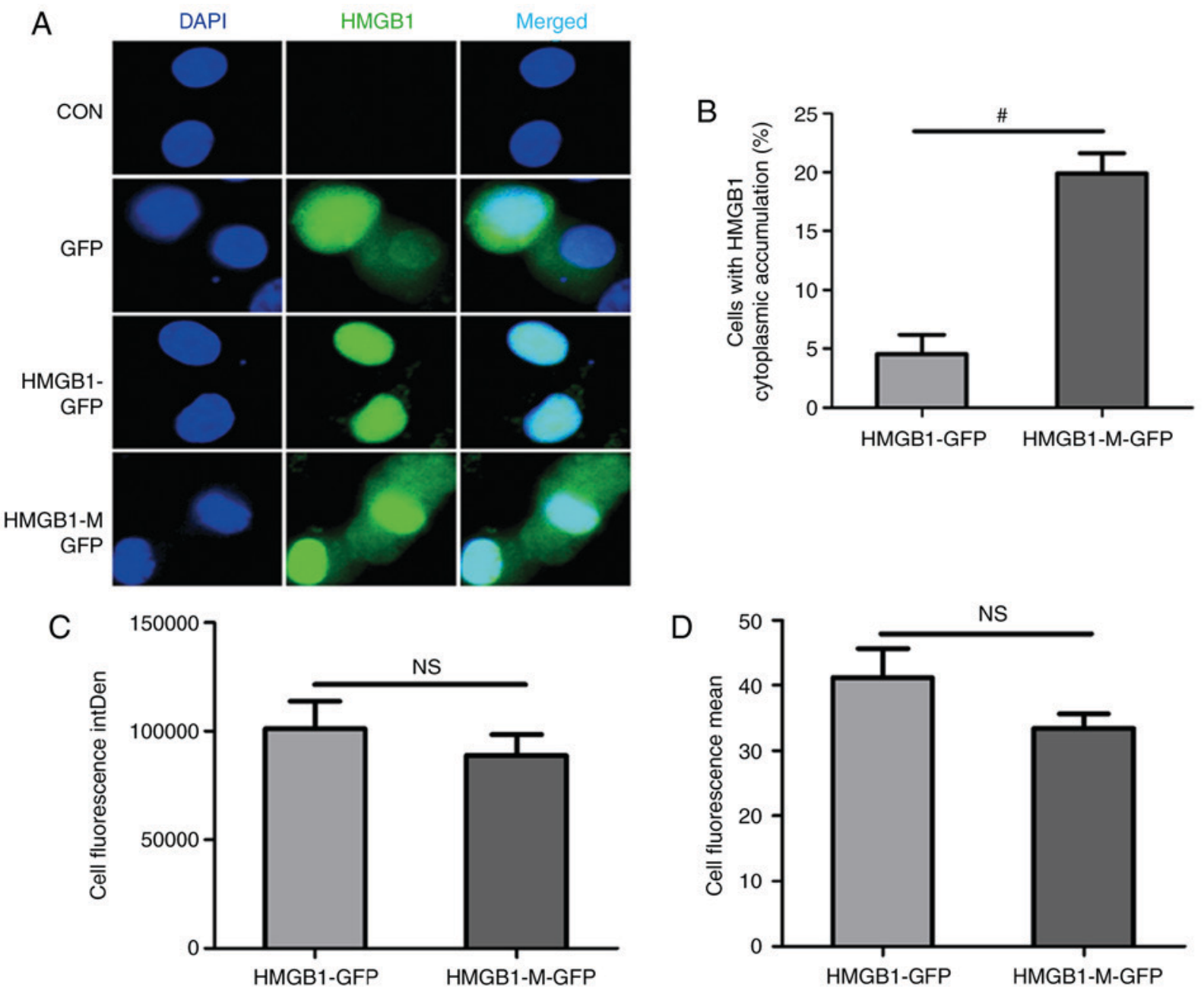

Figure 2. Location of HMGB1 and HMGB1-M in RAW 264.7 cells. (A) Mouse macrophage-like RAW 264.7 cells were transfected without plasmid (CON) or with plasmids [pEGFP-N1 (GFP), pEGFP-N1-HMGB1 (HMGB1-GFP) or pEGFP-N1-HMGB1-M (HMGB1-M-GFP)] for 48 h. Nuclear staining was performed with DAPI (blue) and the localization of the HMGB1 protein (green color) was observed by fluorescence microscopy (magnification, $\mathrm{x} 400$ ). (B) Cells with cytoplasmic accumulation were quantified; data were presented as the mean \pm standard error of the mean of two independent experiments, ${ }^{\sharp} \mathrm{P}<0.05$. (C and D) IntDen and mean fluorescence intensity of HMGB1-M or HMGB1 expression in cells. GFP, green fluorescent protein; HMGB1, high mobility group box 1; HMGB1-M, mimicked acetylated HMGB1; IntDen, integrated; NS, not significant.

the nucleus (Fig. 2A). Compared with HMGB1, HMGB1-M expression significantly increased the percentage of HMGB1 cytoplasmic accumulation in RAW 264.7 cells (Fig. 2B). The integrated (Fig. 2C) and mean fluorescence intensity (Fig. 2D) between the two groups were not statistically significant.

HMGB1-M increases TNF- $\alpha$ production in RAW 264.7 cells. To determine the potential role of HMGB1-M in regulating the functions of macrophages, the phagocytotic function and TNF- $\alpha$ secretion of RAW 264.7 cells treated with HMGB1-M or HMGB1 were investigated. FITC-dextran was phagocytosed by RAW 264.7 cells and did not adhere to its surface (Fig. 3A). HMGB1-M and HMGB1 significantly impaired the phagocytic activity of RAW 264.7 cells compared with that in the MED group (Fig. 3B and C); a significant decrease in the mean fluorescence intensity (MFI) of FITC-dextran phagocytosis in RAW 264.7 cells was observed in the HMGB1-M group compared with in the MED group (Fig. 3D). In addition, HMGB1 and HMGB1-M were also significant inducers of TNF- $\alpha$ production in RAW 264.7 cells compared with in the
MED group. Furthermore, HMGB1-M significantly increased the production of TNF- $\alpha$ in RAW 264.7 cells than HMGB1 (Fig. 3E). These results demonstrated that HMGB1-M increased TNF- $\alpha$ production within RAW 264.7 cells and exerted no significant effects on the phagocytic potential of macrophages.

HMGB1-M decreases phenotypic maturation of BMDCs. The present study evaluated the role of HMGB1-M in BMDC function. HMGB1-M and HMGB1 was applied respectively to stimulate immature BMDC for $48 \mathrm{~h}$. The frequency of CD11c ${ }^{+}$ cells among the total mononuclear cells and percentage of CD11c+ cells expressing CD80, MHC-II or CXCR4 were evaluated. Compared with the MED group, HMGB1-M and HMGB1 increased the expression frequency of MHC-II and CD80 on BMDCs; however, the frequency of CD11c expression was notably unaltered (Fig. 4A-C). Additionally, the present study measured the expression of CXCR4 on BMDCs following stimulation (Fig. 4D). Compared with HMGB1, HMGB1-M significantly decreased the MFI of CD11c but not the percentage of $\mathrm{CD} 11 \mathrm{c}^{+}$cells (Fig. 4E); the percentage 
A

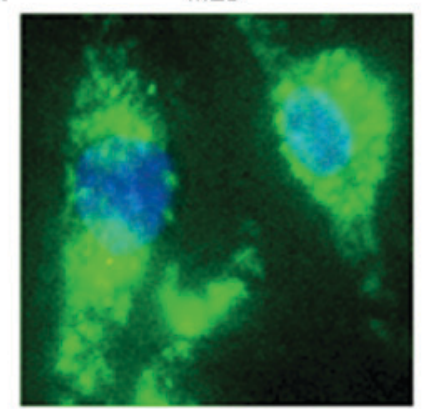

HMGB1-M

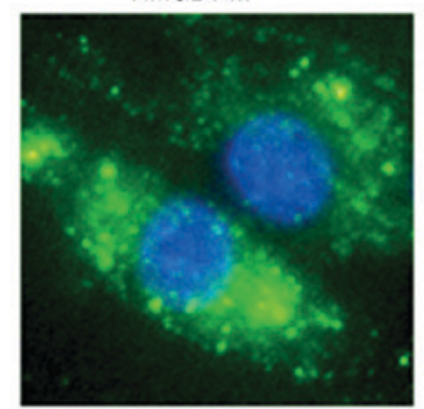

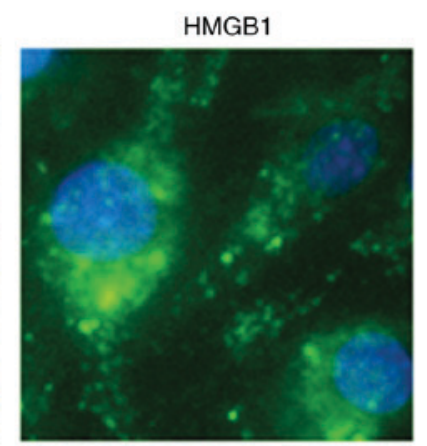

B
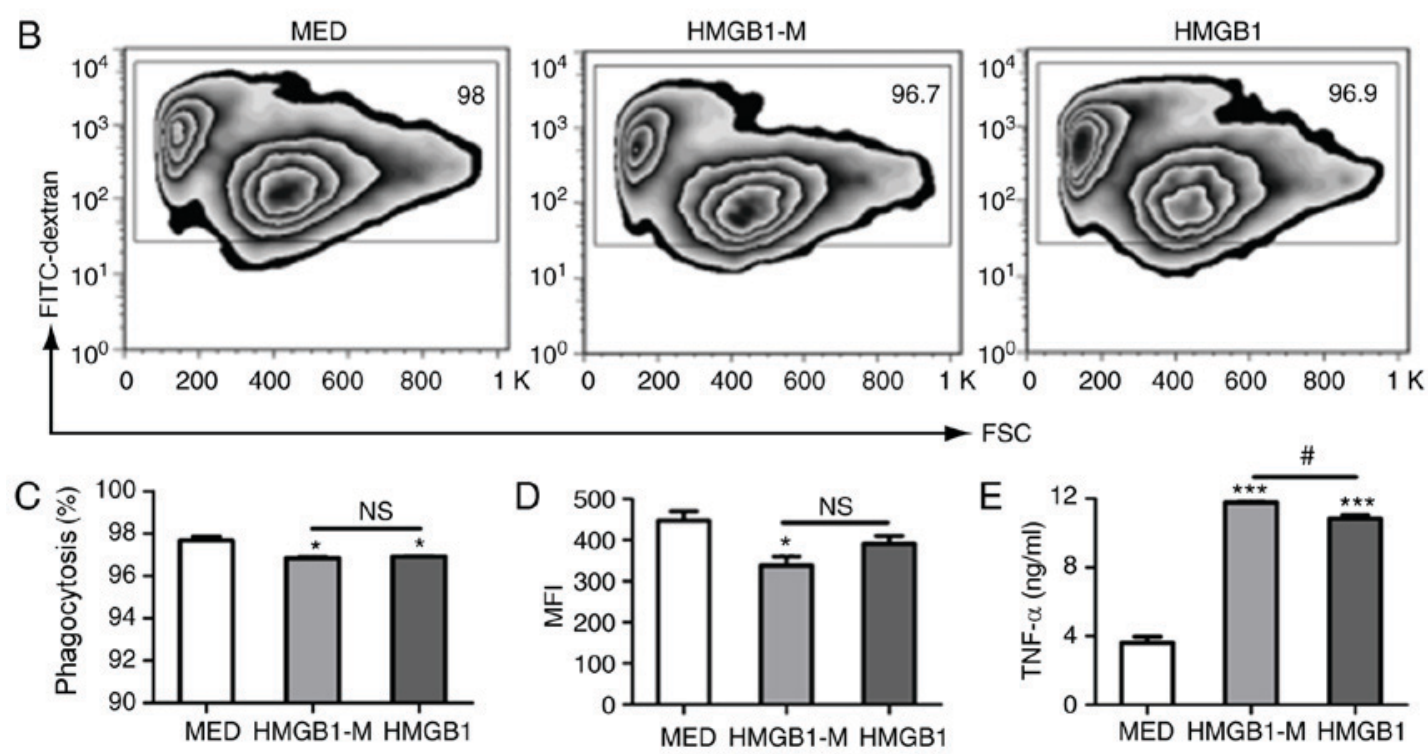

Figure 3. HMGB1-M increases TNF- $\alpha$ secretion in RAW 264.7 cells. The cells were stimulated for $16 \mathrm{~h}$ with HMGB1-M (5 $\mu \mathrm{g} / \mathrm{ml})$ or HMGB1 (5 $\mu \mathrm{g} / \mathrm{ml})$. Following the collection of culture supernatants, the cells were incubated with FITC-dextran for $2 \mathrm{~h}$. (A) Morphological appearances of RAW 264.7 cells phagocytosing FITC-dextran were assessed by fluorescence microscopy. Magnification, x400. (B) Phagocytic activity was analyzed by flow cytometry. (C) Phagocytic ratio of FITC ${ }^{+}$cells. (D) MFI of cells is indicated. (E) TNF- $\alpha$ concentrations in culture supernatants were measured by ELISA. "P $<0.05$, ${ }^{* * * *} \mathrm{P}<0.001$ vs. MED, and ${ }^{*} \mathrm{P}<0.05$. The data are representative of three independent experiments. FITC, fluorescein isothiocyanate; HMGB1, high mobility group box 1; HMBG1-M, mimicked acetylated HMGB1; MED, media control; MFI, mean fluorescence intensity; NS, not significant; TNF- $\alpha$, tumor- $\alpha$.

and MFI of MHC-II-expressing BMDCs was significantly decreased with HMGB1-M compared with HMGB (Fig. 4F). HMGB1-M exhibited no significant effects on the expression of CD80 compared with HMGB (Fig. 4G). It has been demonstrated that HMGB1 is required for the migration of maturing DCs due to upregulation of CXCR4 expression on BMDCs (21). Compared with the MED group, HMGB1-M and HMGB1 significantly promoted the percentage of cells expressing CXCR4; however, no significant differences were found between HMGB1-M and HMGB1 in the expression of CXCR4 on BMDCs (Fig. 4H). These results demonstrated that HMGB1-M downregulated phenotypic maturation of BMDCs as demonstrated by the decreased MHC-II expression; however, no notable effects on the expression of CD80 and CXCR4 on BMDCs were observed.

\section{Discussion}

In the present study, a HMGB1-M plasmid was successfully constructed to mimic the acetylated lysine residues of HMGB1. HMGB1, as an extracellular mediator, serves an important role within macrophages and DCs to facilitate the immune response $(9,10)$. HMGB1-M, compared with HMGB1, increased TNF- $\alpha$ production within RAW 264.7 cells and suppressed BMDC maturation, which may respectively affect the responses to inflammation and antigen presentation.

Point mutation technology is a universal research method for studying the function of proteins (22). To obtain the HMGB1-M protein, the pET28a-HMGB1-M plasmid was constructed, which expressed the HMGB1-M protein with mutated lysine residues, which were converted into glutamines at positions 27, 28, 29, 181, 182 and 183. This mutation process involved a total of six point mutations, in which three mutations were conducted each time. Of note, the final PCR products were inevitably mixed with some parental strands and undesired mutations following DpnI digestion. A few modifications based on other mutation methods were performed in the present study (23-25), which included reducing the concentration of the parent template at the beginning of the PCR mutation reaction and constructing a pair of identification primers to perform the preliminary screening of mutants following site-directed mutagenesis via PCR. The forward primer contained three mutant base sites, with the first base at the 3 ' terminus starting from the mutated site, and the first base at the 5 ' terminus of the reverse primer, which started 

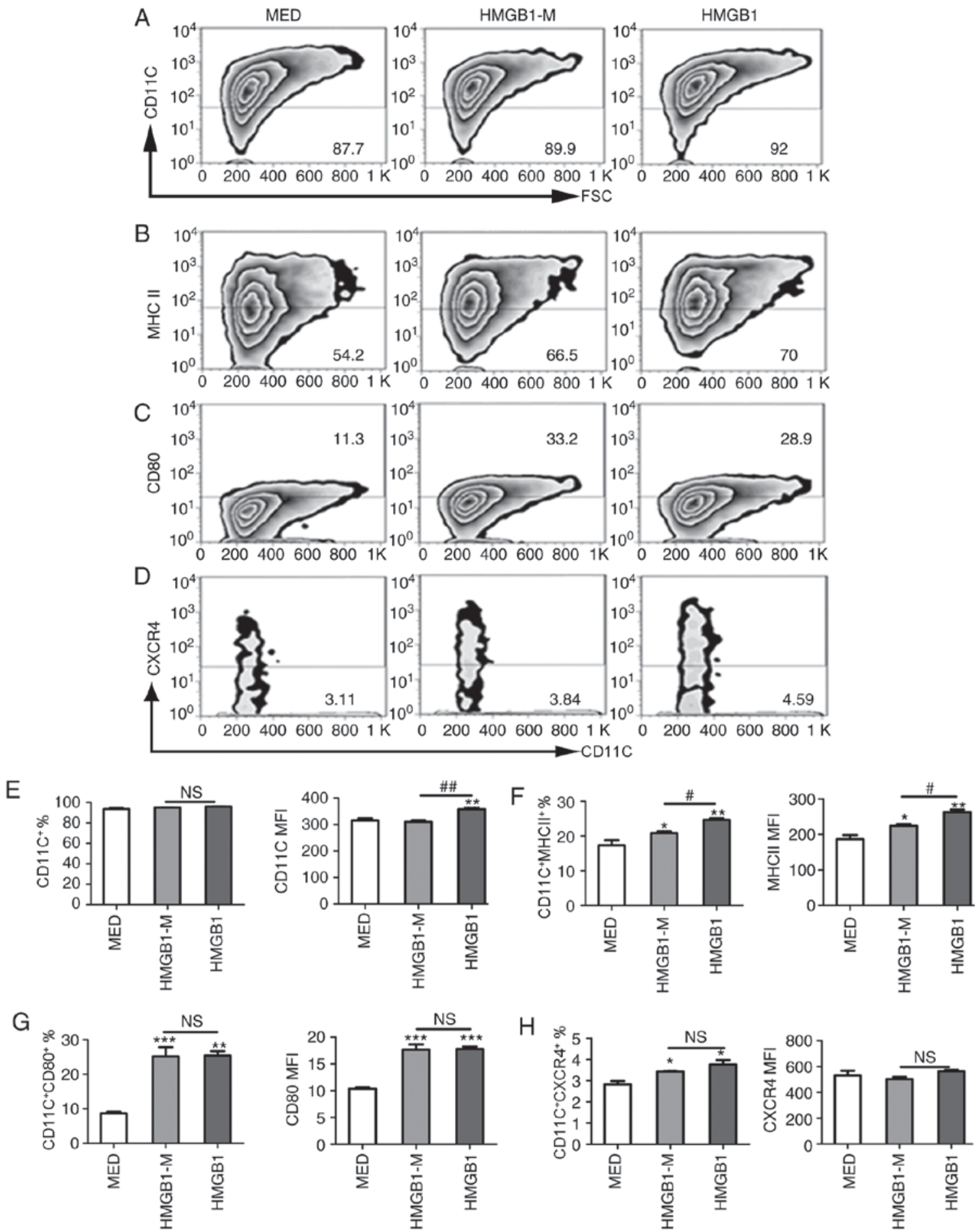

Figure 4. HMGB1-M suppresses phenotypic maturation of BMDCs. The cells were treated with HMGB1-M $(5 \mu \mathrm{g} / \mathrm{ml}) \mathrm{or} \mathrm{HMGB} 1(5 \mu \mathrm{g} / \mathrm{ml})$ for $48 \mathrm{~h}$. (A-D) BMDCs were collected and surface molecule expression was assessed by flow cytometry. (E-H) Frequency of CD11 $\mathrm{c}^{+}$among the total mononuclear cells and percentage of CD80, MHC-II or CXCR4 expression of CD11c+ subsets (left) and MFI is indicated (right). ${ }^{*} \mathrm{P}<0.05,{ }^{* * *} \mathrm{P}<0.01$ and ${ }^{* * * *} \mathrm{P}<0.001 \mathrm{vs}$. $\mathrm{MED}$, and ${ }^{\#} \mathrm{P}<0.05,{ }^{\# \#} \mathrm{P}<0.01$. The data are representative of three independent experiments. BMDCs, bone marrow-derived dendritic cells; CD80, cluster of differentiation 80; HMGB1, high mobility group box 1; HMBG1-M, mimicked acetylated HMGB1; MED, media control; MFI, mean fluorescence intensity; NS, not significant.

from base 335 in the HMGB1 coding sequence. The pair of identifying primers had the following characteristics: At least one of the forward and reverse primers contained three point mutation sites at the $3^{\prime}$ terminus. These optimizations for mutation may aid the generation of mutations in future investigations.
The modification of acetylated HMGB1 is important for its subcellular relocation and release into the extracellular space (4). The two major clusters (NLS1 and NLS2) of HMGB1 lysine residues are regions of high acetylation. Lysine hyperacetylation within two major clusters was reported to affect the subcellular relocation of HMGB1 (15). The mutation 
of six lysine residues (positions: 27, 28, 29, 181, 182 and 183) into glutamines within two major clusters, which mimicked acetylated lysine, was associated with the translocation of HMGB1 from the nucleus to the cytoplasm (15). HMGB1-M acquired by mutation does not possess lysine acetylation in the HMGB1 protein via post-translational modifications (15). Treatment with histone deacetylase inhibitors was reported to increase HMGB1 translocation to the cytoplasm (15). These data are consistent with the results of the present study, which demonstrated that the HMGB1-M protein with mutations of lysine residues at positions 27, 28, 29, 181, 182 and 183 into glutamines was associated with increased cytoplasmic localization of HMGB1-M in RAW 264.7 cells than the HMGB1 protein. In addition, the molecular weight of the HMGB1-M protein band was slightly different from that of HMGB1 in the present study, as the molecular weight of substitutable glutamine $(146.1456 \mathrm{~g} / \mathrm{mol})$ is lower than lysine $(146.1888 \mathrm{~g} / \mathrm{mol})$. Therefore, the mimicked multisite-acetylated HMGB1 was selected as an alternative approach to investigate acetylated HMGB1 in the present study to determine its extracellular function.

Previous studies have reported that actively released HMGB1 from macrophages undergoes post-translational modifications, such as acetylation $(15,19)$; however, HMGB1 released from necrotic cells is not post-translationally modified $(4,9,26)$. Therefore, there are likely two forms of HMGB1 (acetylated and nonacetylated HMGB1) present in the extracellular environment. In the present study, the effects of HMGB1-M with on macrophages and DCs were compared with that of HMGB1. Subsequent analyses on TNF- $\alpha$ production and phagocytic potential in RAW 264.7 cells, BMDC maturation, and CXCR4 expression were conducted. Few investigations into the functions of HMGB1-M and HMGB1 were conducted in the present study; however, notable variations were reported. Compared with HMGB1, HMGB1-M increased TNF- $\alpha$ release from RAW 264.7 cells and decreased BMDC maturation, with no marked effects on phagocytic potential of macrophages, or CD80 and CXCR4 expression. These results suggested that HMGB1-M may serve a proinflammatory role, whereas HMGB1 is more likely to serve a role in antigen presentation. The reported variations between HMGB1-M and HMGB1 were notable; but may serve different roles in the regulation of the immune response. Therefore, further investigation into these associations in vivo are required in the future.

In conclusion, the present study employed a simple and efficient method to induce mutations to conduct functional studies of genes and proteins, which may be used in future investigations; identifying primers were generated in the present study to screen mutants. The results of the present study suggested that mimicked acetylation may partly affect the extracellular response of HMGB1 to inflammation and antigen presentation. Therefore, the findings of the present study may provide novel insight into the role of acetylated HMGB1 for the regulation of immune responses in inflammatory diseases.

\section{Acknowledgements}

The authors would like to thank Mr. Bin Zhang, Dr Hongping Ba, Dr Yan Sun, Dr Huijuan Zou, Dr Bingxia Ming, Dr Ming
Gao and Miss Lifeng Shi from the Department of Immunology, School of Basic Medicine, Tongji Medical College, Huazhong University of Science and Technology (Wuhan, China) for their technical assistance with this study.

\section{Funding}

This study was supported by the National Natural Science Foundation of China (NSFC grant nos. 31670876 and 31470852), and the Major State Basic Research Development Program of China (973 Program, grant no. 2013CB530505).

\section{Availability of data and materials}

All data generated or analyzed during this study are included in this published article.

\section{Authors' contributions}

CX was responsible for the concept and design of the study, acquisition of data, analysis and interpretation of data, drafting the article. HX, XY and XP designed the study. TZ and GF interpreted the data. ZF contributed to the concept and design of the study, interpretation of data, and critical revision of the manuscript.

\section{Ethics approval and consent to participate}

The present study was approved by the Animal Care and Use Committee of Tongji Medical College, HUST (Wuhan, China).

\section{Patient consent for publication}

Not applicable.

\section{Competing interests}

The authors declare that they have no competing interests.

\section{References}

1. Ueda T, Chou H, Kawase T, Shirakawa H and Yoshida M: Acidic C-tail of HMGB1 is required for its target binding to nucleosome linker DNA and transcription stimulation. Biochemistry 43: 9901-9908, 2004.

2. Wang H, Bloom O, Zhang M, Vishnubhakat JM, Ombrellino M, Che J, Frazier A, Yang H, Ivanova S, Borovikova L, et al: HMG-1 as a late mediator of endotoxin lethality in mice. Science 285: 248-251, 1999.

3. Lu B, Nakamura T, Inouye K, Li J, Tang Y, Lundbäck P, Valdes-Ferrer SI, Olofsson PS, Kalb T, Roth J, et al: Novel role of PKR in inflammasome activation and HMGB1 release. Nature 488: 670-674, 2012.

4. Zhang Q and Wang Y: HMG modifications and nuclear function. Biochim Biophys Acta 1799: 28-36, 2010.

5. Tang D, Kang R, Livesey KM, Cheh CW, Farkas A, Loughran P, Hoppe G, Bianchi ME, Tracey KJ, Zeh HJ III and Lotze MT: Endogenous HMGB1 regulates autophagy. J Cell Biol 190: 881-892, 2010.

6. Janko C, Filipovic M, Munoz LE, Schorn C, Schett G, Ivanović-Burmazović I and Herrmann M: Redox modulation of HMGB1-related signaling. Antioxid Redox Signal 20: 1075-1085, 2014.

7. Tang D, Kang R, Zeh HJ III and Lotze MT: High-mobility group box 1, oxidative stress, and disease. Antioxid Redox Signal 14: 1315-1335, 2011. 
8. Venereau E, Casalgrandi M, Schiraldi M, Antoine DJ, Cattaneo A, De Marchis F, Liu J, Antonelli A, Preti A, Raeli L, et al: Mutually exclusive redox forms of HMGB1 promote cell recruitment or proinflammatory cytokine release. J Exp Med 209: 1519-1528, 2012.

9. Dumitriu IE, Baruah P, Manfredi AA, Bianchi ME and Rovere-Querini P: HMGB1: Guiding immunity from within. Trends Immunol 26: 381-387, 2005.

10. Messmer D, Yang H, Telusma G, Knoll F, Li J, Messmer B, Tracey KJ and Chiorazzi N: High mobility group box protein 1 An endogenous signal for dendritic cell maturation and Th1 polarization. J Immunol 173: 307-313, 2004.

11. Andersson U and Tracey KJ: HMGB1 is a therapeutic target for sterile inflammation and infection. Annu Rev Immunol 29 : 139-162, 2011.

12. Sterner R, Vidali G and Allfrey VG: Studies of acetylation and deacetylation in high mobility group proteins. Identification of the sites of acetylation in HMG-1. J Biol Chem 254: 11577-11583, 1979.

13. Ugrinova I, Pasheva EA, Armengaud J and Pashev IG: In vivo acetylation of HMG1 protein enhances its binding affinity to distorted DNA structures. Biochemistry 40: 14655-14660, 2001.

14. Alexandrova EA and Beltchev BG: Acetylated HMG1 protein interacts specifically with homologous DNA polymerase alpha in vitro. Biochem Biophys Res Commun 154: 918-927, 1988.

15. Bonaldi T, Talamo F, Scaffidi P, Ferrera D, Porto A, Bachi A, Rubartelli A, Agresti A and Bianchi ME: Monocytic cells hyperacetylate chromatin protein HMGB1 to redirect it towards secretion. EMBO J 22: 5551-5560, 2003.

16. Assenberg R, Webb M, Connolly E, Stott K, Watson M, Hobbs J and Thomas JO: A critical role in structure-specific DNA binding for the acetylatable lysine residues in HMGB1. Biochem J 411: 553-561, 2008.

17. Elenkov I, Pelovsky P, Ugrinova I, Takahashi M and Pasheva E: The DNA binding and bending activities of truncated tail-less HMGB1 protein are differentially affected by lys- 2 and lys- 81 residues and their acetylation. Int J Biol Sci 7: 691-699, 2011.
18. Lange SS, Mitchell DL and Vasquez KM: High mobility group protein B1 enhances DNA repair and chromatin modification after DNA damage. Proc Natl Acad Sci USA 105: 10320-10325, 2008.

19. Lu B, Antoine DJ, Kwan K, Lundbäck $P$, Wähämaa $H$, Schierbeck H, Robinson M, Van Zoelen MA, Yang H, Li J, et al: JAK/STAT1 signaling promotes HMGB1 hyperacetylation and nuclear translocation. Proc Natl Acad Sci USA 111: 3068-3073, 2014.

20. Lutz MB, Kukutsch N, Ogilvie AL, Rössner S, Koch F, Romani N and Schuler G: An advanced culture method for generating large quantities of highly pure dendritic cells from mouse bone marrow. J Immunol Methods 223: 77-92, 1999.

21. Dumitriu IE, Bianchi ME, Bacci M, Manfredi AA and Rovere-Querini P: The secretion of HMGB1 is required for the migration of maturing dendritic cells. J Leukoc Biol 81: 84-91, 2007.

22. Hsieh PC and Vaisvila R: Protein engineering: Single or multiple site-directed mutagenesis. Methods Mol Biol 978: 173-186, 2013.

23. Aiyar A, Xiang Y and Leis J: Site-directed mutagenesis using overlap extension PCR. Methods Mol Biol 57: 177-191, 1996.

24. Zheng L, Baumann U and Reymond JL: An efficient one-step site-directed and site-saturation mutagenesis protocol. Nucleic Acids Res 32: e115, 2004.

25. Liu Y, Wu T, Song J, Chen XL, Zhang Y and Wan Y: A mutant screening method by critical annealing temperature-PCR for site-directed mutagenesis. BMC Biotechnol 13: 21, 2013.

26. Scaffidi P, Misteli T and Bianchi ME: Release of chromatin protein HMGB1 by necrotic cells triggers inflammation. Nature 418: 191-195, 2002.

This work is licensed under a Creative Commons Attribution-NonCommercial-NoDerivatives 4.0 International (CC BY-NC-ND 4.0) License. 